\title{
A Study of Signal Timing Fuzzy Control Based on the Collaborative Strategy of Road Green Wave Effect and Path Selection Entropy
}

\author{
Yi Zhang ${ }^{*}$, Gang Wang and Gengsheng Huang
}

\author{
South China Institute of Software Engineering of GuangZhou University, Guangzhou 510990, China
}

\begin{abstract}
With the growth of urban road traffic volume and the increase of road network density, correlation between adjacent road intersections is becoming more and more obvious. An intersection traffic signal adjustment tends to affect the smooth traffic flow of a number of adjacent intersections. Its congestion may gradually spread to a few blocks and regions all around the intersection. Therefore, increasingly high demands for urban traffic signal control make a variety of advanced control technology integrated, so as to adjust the intersection control parameter and accordingly achieve a dynamic coordination control over the city traffic. Hopefully, it can satisfy traffic demands and achieve a new dynamic balance between road traffic and transport demand. This paper focuses on the fuzzy control over green time extension at adjacent intersections of trunk road by using the collaborative strategy of road green wave effect and path selection entropy, in order to solve the problem of coupling between adjacent intersections, so as to realize the signal coordination control on trunk road. This algorithm makes Signal Timing more flexible.
\end{abstract}

Keywords: Cooperative policies, green-wave effect, path selection entropy, road, signal fuzzy control.

\section{SIGNAL COORDINATE CONTROL OF ROAD}

In this article, we study signal Coordinate Control of adjacent intersections. As showed in Fig. (1). We can model it as undirected graph $\mathrm{G}$ (V.E), in this figure, $\mathrm{V}$ was junctions collection, $\mathrm{E}$ was an intersection between roads collection. In the green wave phenomena, many traffic lights collaborate, makes some direction of traffic through multiple junctions in a row by vehicles to maximize the number of vehicles through the intersection, minimize vehicles waiting at the crossing.

According to the green wave effects of the collaboration for the traffic lights, the number of waiting vehicles in the next time is required to be minimum, that is to say, the expected utility of the value is required to be minimum at $t$ moment of $\lambda$ phase [1].

$$
\pi *(\lambda, t)=\operatorname{argmin}_{\mathrm{J} \_ \text {act }(\mathrm{G}, \lambda, \mathrm{t})} E U(G, \lambda, t) \text {, to consider } \lambda \text { as }
$$

signal phase, according to the change of road right-of-way within a period to divide.

$\mathrm{EU}(\mathrm{G}, \lambda, \mathrm{t})$, refers to the utility value produced by the collaboration working at $\mathrm{t}$ moment from intersection $V_{j}$ to intersection $V_{i}$ and turning to intersection $V_{k}$ will equal to the number of waiting vehicles at each intersection of the entire road at $\mathrm{t}+1$ moments:

$$
E U(G, \lambda, t)=\sum_{\mathrm{v}_{\mathrm{i}} \in V} \sum_{\mathrm{vj} \in n\left(V_{i}\right)} T f_{i}^{j}(k, \lambda, t+1)
$$

*Address correspondence to this author at the South China Institute of Software Engineering of GuangZhou University, Guangzhou 510990, China; Tel: 86-020-87818126; Fax: 86-020-87818127;

E-mail: zy@sise.com.cn
To consider $T f_{i}^{j}(k, \lambda, t+1)$ as $\lambda$ phase of the current lane waiting for $t+1$ moment the number of vehicles, and to consider $J t \_a c t(G, \lambda, t)$ as this is a moment $t \lambda$ phase collection of all the action of traffic lights, that is

$$
\text { Jt_act }(\mathrm{G}, \lambda, \mathrm{t})=\bigcup_{\mathrm{V}_{\mathrm{i}} \in \mathrm{V}} \bigcup_{\mathrm{Vj} \in \mathrm{V}} £_{\mathrm{i}}^{\mathrm{j}}(k, \lambda, t), \quad \text { and } \quad £_{\mathrm{i}}^{\mathrm{j}}(k, \lambda, t) \quad \in
$$

$\{$ green, red $\}$ show the light to change to green and red.

According to the person who name $\mathrm{Xu}$ yang and the other person put The Predictive Model to us, we can calculate the leave and arrival of the number of intersection vehicle, the next moment, $\lambda$ phase the number of vehicles in the driveway equal to the number of vehicles waiting to be minus the left of the number of vehicles and adds the number of vehicles to arrive that is

$T f_{i}^{j}(k, \lambda, t+1)=T f_{i}^{j}(k, \lambda, t)+\mathrm{Tc}_{\mathrm{i}}^{\mathrm{j}}(\mathrm{k}, \lambda, \mathrm{t})-\mathrm{Td}_{\mathrm{i}}^{\mathrm{j}}(\mathrm{k}, \lambda, \mathrm{t})$

To consider $\operatorname{Td}_{i}^{\mathrm{j}}(\mathrm{k}, \lambda, \mathrm{t})$ as $\lambda$ phase of the current lane leaving for $t+1$ moment the number of vehicles: We assume that every lane in a green light cycle through the maximum number of vehicles to $\delta$, if $£_{\mathrm{i}}^{\mathrm{j}}(\mathrm{k}, \lambda, \mathrm{t})=$ green, $\operatorname{Td}_{\mathrm{i}}^{\mathrm{j}}(\mathrm{k}, \lambda, \mathrm{t})=$ $T f_{i}^{j}(k, \lambda, t)<=\delta$; but if $£_{\mathrm{i}}^{\mathrm{j}}(k, \lambda, t)=\mathrm{red}, \operatorname{Td}_{\mathrm{i}}^{\mathrm{j}}(\mathrm{k}, \lambda, \mathrm{t})=0$.

To consider $\operatorname{Tc}_{\mathrm{i}}^{\mathrm{j}}(\mathrm{k}, \lambda, \mathrm{t})$, it means in the current lane in $\lambda$ phase at $\mathrm{t}$ moment, the number of vehicles equals to the number of vehicles in the previous green light cycle which select to enter the lane. Path entropy in the $\lambda$ phase can be used to get the choosing probability $\beta_{\mathrm{i}, \mathrm{j}, \mathrm{k}}$ of vehicles which run from intersection $V_{j}$ ( $m$ position), cross intersection $V_{i}$ and turn to intersection $V_{K}$ ( $n$ position). Entropy is a mathe- 


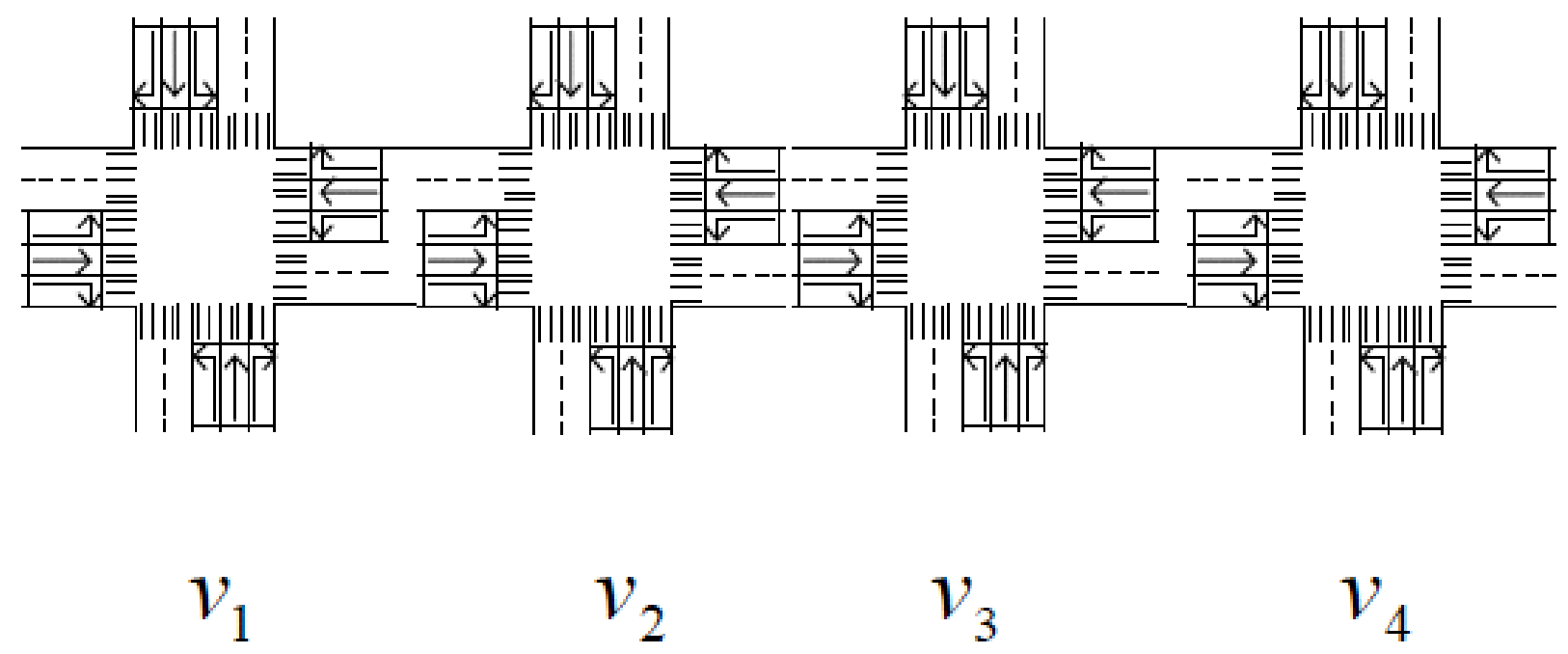

Fig. (1). Adjacent intersections model.

matical measure for information or uncertainty and it can be attained from the calculation of probability distribution.

$\mathrm{Tc}_{\mathrm{i}}^{\mathrm{j}}(\mathrm{k}, \lambda, \mathrm{t})=\beta_{\mathrm{i}, \mathrm{j}, \mathrm{k}} \times \sum_{\mathrm{V}_{1} \in \mathrm{n}\left(\mathrm{V}_{\mathrm{j}}\right) \wedge \mathrm{v}_{\mathrm{1}} \neq \mathrm{v}_{\mathrm{i}}} T d_{j}^{l}(i, \lambda, \mathrm{t})$

In the traffic flow, vehicle position and speed is an important characteristic of its movement. We assume that the characteristic variable of vehicle running from $\mathrm{m}$ position to $\mathrm{n}$ position is $a_{m, n} \cdot a_{m, n}(t)$ refers to the characteristic variable of vehicle at $t$ moment, which becomes $a_{m, n}(t) \rightarrow a_{m, n}\left(t+\Delta_{\mathrm{t}}\right)$ at $\Delta_{\mathrm{t}}$ time interval. $v(m, t)$ and $v(n, t)$ refers to the speed variable at position $m$ and $n$ at $t$ moment respectively, and the relative speed variable can be defined as:

$v(m, n, t)=\mathrm{v}(\mathrm{m}, \mathrm{t})-\mathrm{v}(\mathrm{n}, \mathrm{t})$

$p(m, t)$ and $p(n, t)$ refers to the position variable at position $m$ and $n$ at $t$ moment respectively, and the relative position variable can be defined as:

$p(m, n, t)=p(\mathrm{~m}, \mathrm{t})-p(\mathrm{n}, \mathrm{t})$

Thus, the characteristic variable of vehicle running from position $m$ to $n$ is $a_{m, n}$, which can be defined as:

$a_{m, n}=\frac{1}{\mathrm{~N}} \sum_{\mathrm{i}=1}^{\mathrm{N}} \frac{\left|p\left(m, n, t_{i}\right)+\mathrm{v}\left(\mathrm{m}, \mathrm{n}, \mathrm{t}_{\mathrm{i}}\right) \times \Delta_{\mathrm{t}_{\mathrm{i}}}\right|-\left|p\left(\mathrm{~m}, \mathrm{n}, \mathrm{t}_{\mathrm{i}+1}\right)\right|}{R}$

In this formula, $N$ is the number of discrete time $t_{i}$ at $\Delta_{t}$ time interval, namely, the times of the relative position variable and the relative speed variable being calculated and updated at every $\Delta$ time interval. $R$ refers to vehicle distance. Path entropy $H_{m}\left(t, \Delta_{\mathrm{t}}\right)$ can be defined as:

$H_{m}\left(t, \Delta_{\mathrm{t}}\right)=\frac{-\sum_{\mathrm{k} \in \mathrm{F}_{\mathrm{m}}} P_{k}\left(t, \Delta_{\mathrm{t}}\right) \log P_{k}\left(t, \Delta_{\mathrm{t}}\right)}{\log \mathrm{C}\left(\mathrm{F}_{\mathrm{m}}\right)}$
Here in $P_{k}\left(t, \Delta_{\mathrm{t}}\right)=\frac{\mathrm{a}_{\mathrm{m}, \mathrm{k}}}{\sum_{i \in \mathrm{F}_{\mathrm{m}}} a_{m, i}}, F_{m}$ refers to the position set of the relative position variable being calculated and updated at every $\Delta$ time interval. $C\left(F_{m}\right)$ refers to the comparability of set $F_{m}$, and $0 \leq \mathrm{H}_{\mathrm{m}}\left(\mathrm{t}, \Delta_{\mathrm{t}}\right) \leq 1$. From the definition of path entropy $H_{m}\left(t, \Delta_{\mathrm{t}}\right)$, it can be found that the more frequently the vehicle movement change, the smaller the entropy $H_{m}\left(t, \Delta_{\mathrm{t}}\right)$ change. Based on the above definition, $\beta_{\mathrm{i}, \mathrm{j}, \mathrm{k}}$ can be attained from path entropy $H_{m}\left(t, \Delta_{\mathrm{t}}\right)$.

$[2,3]$ Signal cycle generally should not exceed $200 \mathrm{~s}$ because too long cycle will lead drivers to irritable, thus increase the likelihood of running the red light or imposed to adjust to another line.

\section{CALCULATE ADJACENT JUNCTIONS $V_{i}$ SIGNAL WITH FUZZY CONTROL THOUGHT}

(1) set the time of minimum green light to each phase $G_{\min }=15 \mathrm{~s}$;

(2) when control start, the moment of the $\lambda$ phase of green light to the end and the yellow light begins to flash, according to the strategy of $\pi *(\lambda, t)$, to obtain the number of the $\lambda+1$ phase waiting vehicles and the number of the $\lambda+2$ phase waiting vehicles;

(3) calculate the difference between the number of the $\lambda+2$ phase waiting vehicles and the number of the $\lambda+1$ phase waiting vehicles and credited as $\mathrm{X}$, as well as the difference between the number of vehicles pull into the adjacent intersection and the number of vehicles put out of it and credited as $Y=\mathrm{Tc}_{\mathrm{i}}^{\mathrm{j}}(\mathrm{k}, \lambda, \mathrm{t})-\mathrm{Td}_{\mathrm{i}}^{\mathrm{j}}(\mathrm{k}, \lambda, \mathrm{t})$, get fuzzy control of the two inputs;

(4) look-up the procedures to get the green light delay GE, resulting in the time of phase green light $G_{\text {time }}=G_{\min }+\mathrm{GE}$; 
Table 1. The membership function of $X$.

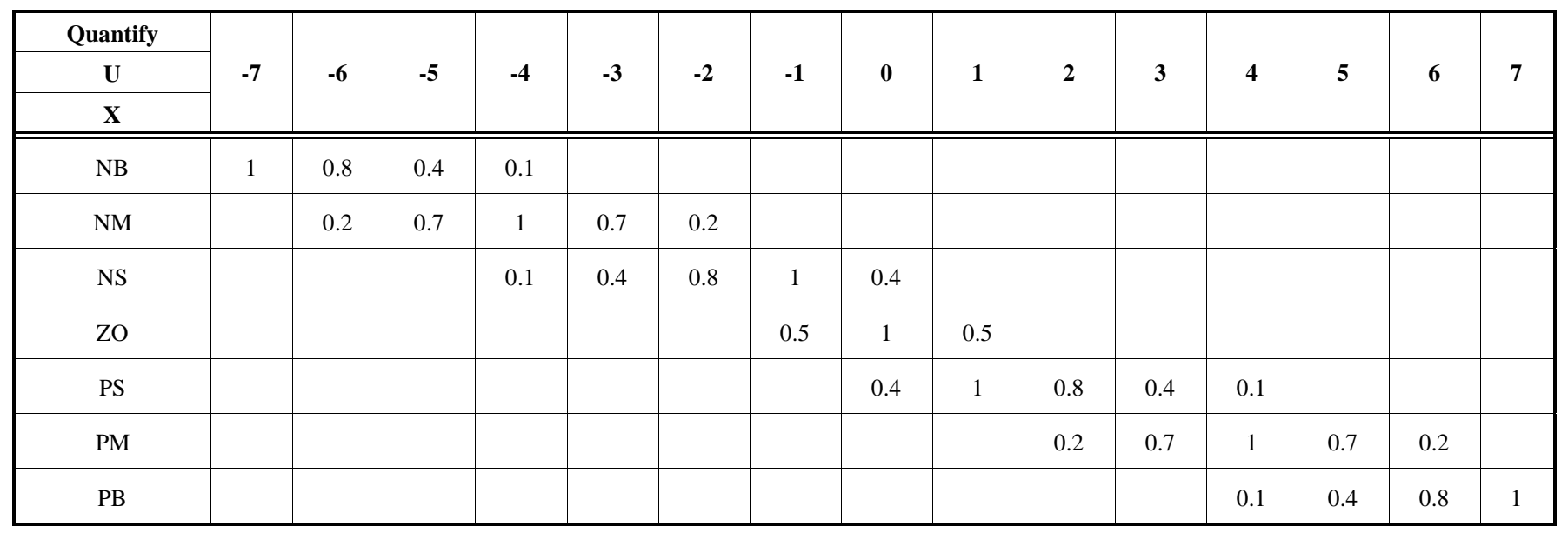

Table 2. Membership function $u$.

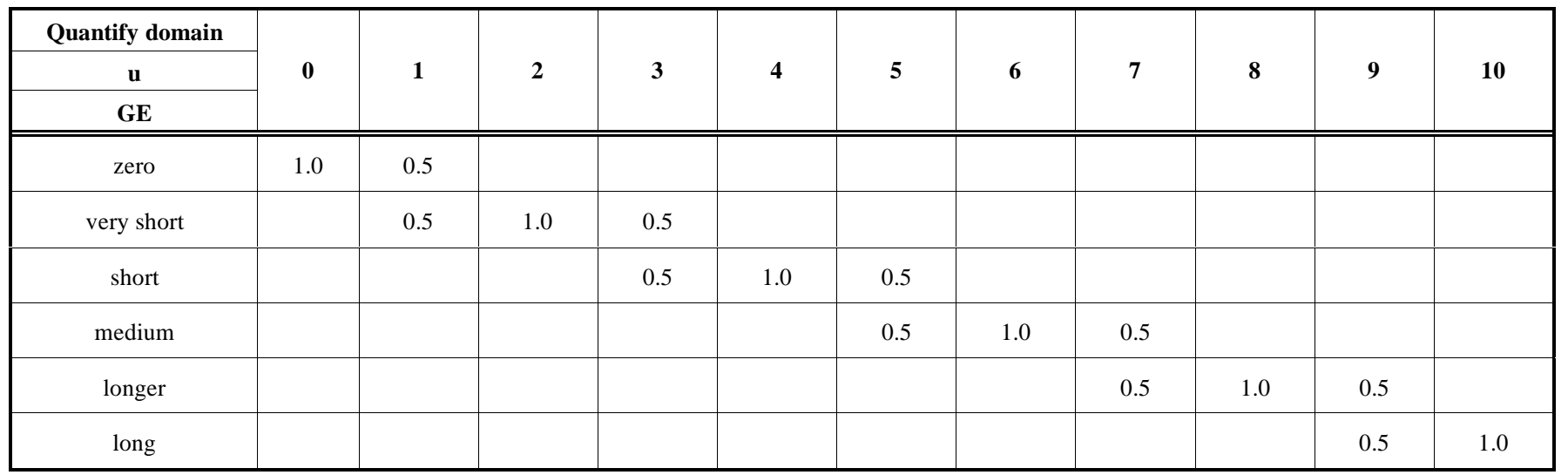

(5) After the $\lambda$ phase red light brightens, the $\lambda+1$ phase yellow light brightens and green light brightens 3 s later, control the green time according to the Gtime got from the table in the last step;

(6) When the time of the $\lambda+1$ phase green light ends and the yellow light brightens, begin to receiving the next phase data, thereby the signal's intelligent controls accomplished.

\subsection{Input Parameters $X, Y$ of Fuzzification [4-6]}

(1) According to the actual situation, determine the actual variation range(basic domain) of $\mathrm{X}$ and $\mathrm{Y},[-30,-30]$ and $[-60,60]$ respectively. Discretization of the basic domain in accordance with the general canonical form: $\{-n,-(n-1), \ldots,-$ $1,0,1, \ldots,(\mathrm{n}-1)$, and $\mathrm{n}\}$. When use the fuzzy control, generally do not target high control accuracy, therefore on the discrete point $\mathrm{s}$ in the domain is not too large. According to the requirement of control accuracy to be achieved, select $n$ is 7 , that is the quantization on domains are: $\{-7,-6,-5,-4,-3$, $-2,-1,0,1,2,3,4,5,6,7\}$. The relationship between discrete values and the input exact value can be obtained according to the quantization factor:

$\mathrm{X}$ discrete value $=$ INT the $\left(K_{x} \times\right.$ actual input to the precise amount+0.5) $K_{x}=7 / 30$ (quantization factor); Y discrete value $=$ INT $\left(K_{y} \times\right.$ actual input to the exact amount +0.5$)$ $K_{y}=7 / 60$ (quantization factor)
(2) The rules of choosing the linguistic variables for fuzzy control should take the characters of flexibility and easy control into consideration, we can select: NB (negative big), NM (negative medium), NS(negative small) ZO (zero), PS (positive small), PM(positive median), PB (positive big). $[7,8]$ the linguistic variables of domain fuzzy subset described by the membership function, the membership function u can determine by summarize the expertise or statistical methods. The membership function of $X$ shown in Table $\mathbf{1}$, the membership function of $\mathrm{Y}$ is the same as $\mathrm{X}$.

\subsection{Output (Green Light Delay) GE Fuzzification}

(1) Its basic domain in [0,50], consider the green light delay only has the positive, the quantization domain is: $\{0,1,2,3,4,5,6,7,8,9,10\}$. Follow the scale factor to get their relationship between discrete values and the exact amount of the actual output:

The actual output of the exact amount $=K_{u} \times$ discrete values

$K_{u}=50 / 10 \quad$ (scale factor)

(2) Fuzzy control's language variables and variables election: zero $\left(G_{0}\right)$, very $\operatorname{short}\left(G_{1}\right), \operatorname{short}\left(G_{2}\right), \operatorname{medium}\left(G_{3}\right)$, longer $\left(G_{4}\right)$, long $\left(G_{5}\right)$. Membership function $u$ shown in Table 2. 
Table 3. Fuzzy control rules.

\begin{tabular}{|c|c|c|c|c|c|c|c|}
\hline $\mathbf{X}$ & \multirow{3}{*}{ NB } & \multirow{3}{*}{ NM } & \multirow{3}{*}{ NS } & \multirow{3}{*}{ ZO } & \multirow{3}{*}{ PS } & \multirow{3}{*}{ PM } & \multirow{3}{*}{ PB } \\
\hline GE & & & & & & & \\
\hline $\mathbf{Y}$ & & & & & & & \\
\hline $\mathrm{NB}$ & zero & zero & zero & zero & zero & zero & zero \\
\hline NM & zero & zero & zero & zero & zero & zero & Very short \\
\hline NS & zero & zero & zero & zero & zero & Very short & Short \\
\hline $\mathrm{ZO}$ & zero & zero & zero & zero & Very short & Short & Medium \\
\hline PS & zero & zero & zero & Very short & Short & Medium & Longer \\
\hline PM & zero & zero & Very short & Short & Medium & Longer & Long \\
\hline $\mathrm{PB}$ & zero & Very short & Short & Medium & Longer & Long & Long \\
\hline
\end{tabular}

\subsection{Fuzzy Control Rule Table Creation}

The establishment of fuzzy control rule is very important, whether the rules can correctly reflect the expert experience and knowledge, whether it can effectively control the object, is directly related to the performance of the controller. Control experience-based summary 49 control rules are as follows Table 3.

\subsection{Fuzzy Inference Algorithm Defuzzification}

In general, the results obtained from the fuzzy rules are still the fuzzy quantities, and the accurate quantities can only be output after reduced by fuzzy reasoning algorithm. According to the fuzzy synthesis reasoning rules, the fuzzy control rules are a synthesis of the output of a fuzzy set:

For example: if $X_{i}$ and $Y_{j}$ then $G E_{m}$

$(i, j$ in [NM, NS ,NB, ZO, PS, PM, PB]; $m$ in [zero, very short, short, medium, longer, long]

The fuzzy relation:

$$
\tilde{R}=\left[X_{i} \times Y_{j}\right]^{T}{ }^{1} \times G E_{m}
$$

Wherein $T_{1}$ represents the relationship between a matrix operation, the purpose is to a $15 \times 15$-dimensional matrix transpose into a $225 \times 1$-dimensional column vector, and finally get a $225 \times 11$-dimensional matrix. Each fuzzy relationship matrix is $225 \times 11$ dimensions, a total of 49 .

Assume that the input variable $X$ is 3 . Membership function maximum value corresponding to the interval PM, let $\mathrm{a}=(0,0,0,0,0,0,0,0,0,0.2,0.7,1,0.7,0.2,0)$; input variable $\mathrm{Y}$ is -2 , membership function maximum value corresponding to the interval of $\mathrm{NS} b=(0,0,0,0.1,0.4,0.8,1,0.4,0,0,0,0,0,0,0)$. According to the fuzzy synthetic arithmetic calculation: According to the fuzzy synthetic arithmetic calculation:

$$
C=(\mathrm{a} \times \mathrm{b})^{\mathrm{T}}{ }^{2} \circ \tilde{R}
$$

Wherein $T_{2}$ represents a matrix operation, the purpose is the matrix transpose of a $15 \times 15$ dimensional row vector dimension into a $1 \times 225$, for the fuzzy rules: if $X=\mathrm{PM}$ and $Y=$ NS then $G E=G_{1}$ Fuzzy relationship matrix.

Eventually get to a $1 \times 11$ dimensional vector. Fuzzy weighted average method is more commonly used, has good stability, its output is continuous but not jumping.

Assuming the output $\mathrm{c}$ is: $\left(c_{1}, c_{2}, c_{3}, c_{4}, c_{5}, c_{6}, c_{7}, c_{8}, c_{9}\right.$, $\left.c_{10}, c_{11}\right)$, each of which an $c_{i}$ value [0,1]. It is the weighted average of the green light time delay:

$G E=\sum\left(\mathrm{i} \times \mathrm{C}_{\mathrm{i}}\right) / C_{i}$ i in $[1-11]$

The final phase o the effective green time: $G_{\text {time }}=G_{\min }+$ GE. After conversion, the green light in the other direction, and repeat the process.

\section{THE TIMING DESIGN OF ROAD SIGNAL COOR- DINATION CONTROL}

The timing scheme of the road coordination control system is usually described by time - distance diagram $[9,10]$. As showed in Fig. (2), Time (signal timing) is the vertical axis, and the distance between intersections on the road is $\mathrm{x}$ axis.

The above adjacent intersection $V_{i}$ signal timing fuzzy control is used to determine the cycle duration of every intersection, and the maximum is used as public cycle duration, that is: $C_{m}=\max \left(\mathrm{C}_{1}, \mathrm{C}_{2}, \ldots, \mathrm{C}_{\mathrm{j}}, \ldots\right)$, with $C_{m}$ as public cycle duration and $C_{j}$ as the cycle duration of intersection $j$. Based on the road coordination, the calculation of green light signal ratio is divided into key intersection and non-critical intersection, and the formula is as follows:

$t_{E G_{m}}=\left(\mathrm{C}_{\mathrm{m}}-\mathrm{L}_{\mathrm{m}}\right) \times \frac{\mathrm{y}_{\mathrm{m}}}{\mathrm{Y}_{\mathrm{m}}}$

In the formula, $t_{E G_{m}}$ refers to the shortest time of the coordinate phase at key intersections, and $C_{m}$ refers to public cycle duration, and $L_{m}$ refers to the total loss time at key 


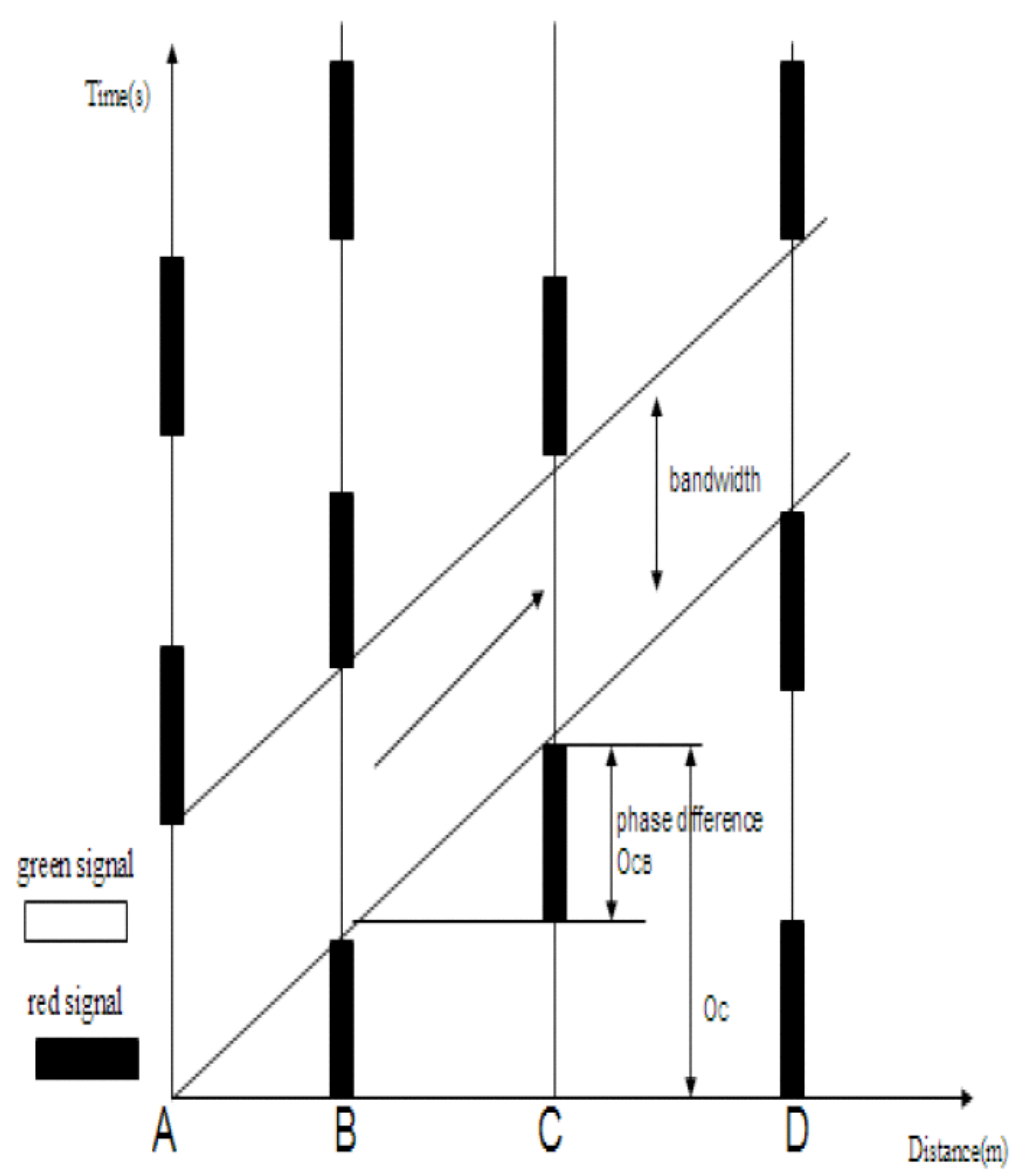

Fig. (2). Time - distance diagram.

intersections. $y_{m}$ refers to the key traffic flow ratio of coordinate phase at key intersections. $Y_{m}$ refers to the sum of the key traffic flow of coordinate phase at key intersections.

$t_{E G_{n}}=\frac{\mathrm{C}_{\mathrm{m}} q_{\mathrm{n}}}{S_{n} \chi_{\mathrm{p}}}=\frac{\mathrm{C}_{\mathrm{m}} y_{n}}{\chi_{\mathrm{p}}}$

In the formula, $t_{E G_{n}}$ refers to the shortest effective green time of the number $n$ phase in non-coordination phases at non-critical intersections. $q_{n}$ refers to the key traffic flow of the number $n$ phase in non-coordination phases at noncritical intersections. $S_{n}$ refers to the saturated flow of key traffic lane in the number $n$ phase of non-coordination phase at non-critical intersections. $\chi_{\mathrm{p}}$ refers to the practical value of the non-coordination phase saturation at non-critical intersections, generally take the value as $0.9 ; y_{n}=\frac{\mathrm{q}_{\mathrm{n}}}{\mathrm{S}_{\mathrm{n}}}$ refers to the ratio of key traffic flows in the number $n$ phase of noncoordination phase at non-critical intersections.

$t_{E G}=\mathrm{C}_{\mathrm{m}}-\mathrm{L}-\sum_{\mathrm{n}=1}^{\mathrm{k}} t_{E G_{n}}$

In the formula, $t_{E G}$ refers to the effective green time of the coordination phases at non-critical intersections. $L$ refers to the total loss time at non-critical intersections. $k$ refers to the total phase of non-coordination phases at non-critical intersections.

According to the above calculation steps, the effective green time of each phase at each intersection can be worked out, and a unified calculation of the green light display time at each phase can be made. Finally, graphical method or algebraic method can be used to calculate phase difference, which may directly affect the control effect of the system. Through geometric mapping, graphical method uses time distance diagram which reflects traffic movement to preliminary build an interactive or synchronous coordination system. Through repeated adjustment based on the belt speed and cycle duration, the phase difference can be decided and finally an ideal green wave belt (pass belt) can be worked out. Algebraic method looks for the minimum quantity of the biggest gap between the actual signal position in each system and the ideal signal position, so as to obtain a best control scheme of phase difference.

\section{CONCLUSION}

Based on the green wave effect and path selection entropy, the use of fuzzy control methods, such as artificial neural networks technology traffic light control system for global optimization scheduling system. Local fuzzy control strategy must follow the principles of the global optimum. We can use the Markov decision process to select the optimal control behavior. The fuzzy control strategy is a composite strategy. It can be adjusted under the control of the 
control center. Entry a lot of fuzzy Data, to train the neural network in order to achieve global optimization scheduling. Although there are still many problems in the global regulation and local control with the use of fuzzy pending to resolve, but as the studies continue, the method will be a good way to solve the problem of Small and Medium Cities in China urban traffic control.

Take control of a variety of advanced technologies integrated with each other to achieve the purpose of intersection adjusts a control parameter, in order to achieve dynamic coordination of traffic throughout the city-wide control, meet the need of traffic demands, let traffic roads and traffic needs reach new dynamic balance, build a scientific, system control system for urban traffic.

\section{CONFLICT OF INTEREST}

The authors confirm that this article content has no conflicts of interest.

\section{ACKNOWLEDGEMENTS}

Declared none.

\section{REFERENCES}

[1] X. Yang, "Agent-based decentralized cooperative traffic control toward green-waved effects,"J. Softw., vol. 23, no. 11, pp. 29392941, Nov. 2012.

[2] L.L. Xi, "Control signal coordination of two adjacent traffic intersections," Acta Automatica Sinica, vol. 29, no. 6, pp. 947-948, Nov. 2003.

[3] G.J. Xia, "Probe into fuzzy control and simulation in multi-phase isolated intersection," Computer and Communications, vol. 22, no. 2, pp. 84-86, Apr. 2004.

[4] L. Jing, "The fuzzy algorithm control for Single road intersection traffic light and its simulation," Microcomputer Information, vol. 3, no. 1, pp. 33-35, Oct. 2006.

[5] C. Wei, "A study on traffic control of signalized intersection based on fuzzy logic," J. Kunming University of Science and Technol., vol. 29, no. 3, pp. 98-100, Jun. 2004.

[6] S. Guo, "Multi-phase fuzzy traffic control based on cyclicmajorization," Decision-making and Control, vol. 17, pp. 14-20, Jun. 2002.

[7] Y. Deng, "To realize fuzzy control of crossroad traffic lights use PLC,"J. Chengdu University, vol. 29, no. 6, pp. 17-18, Feb. 2001.

[8] J. Min, "Fuzzy control and simulation of the urban trunk traffic Signal," J. Dongnan University, vol. 24, no. 4, pp. 29-30, 2008.

[9] B. Sun, C. Gui, Q. Zhang, B. Yan, W. Liu, "A multipath ondemand routing with path selection entropy for ad hoc networks," J. Softw., vol.19, no. zk, pp. 112-120, Dec. 2008.

[10] X. Guo, "Analysis basic of road traffic", CN: Press of Dongnan University, 2010, pp. 335-340.

(C) Zhang et al.; Licensee Bentham Open.

This is an open access article licensed under the terms of the Creative Commons Attribution Non-Commercial License (http://creativecommons.org/licenses/by-nc/3.0/) which permits unrestricted, non-commercial use, distribution and reproduction in any medium, provided the work is properly cited. 